\title{
Anemia as a Problem: GP Approach
}

\author{
Tamara Milovanovic ${ }^{a, b}$ Sanja Dragasevic ${ }^{a, b}$ Andreja Nebojsa Nikolic ${ }^{a}$ \\ Aleksandra Pavlovic Markovic ${ }^{a, b}$ Milica Stojkovic Lalosevic ${ }^{a, b}$ \\ Dusan D. Popovic ${ }^{a, b}$ Miodrag N. Krstic ${ }^{a, b}$

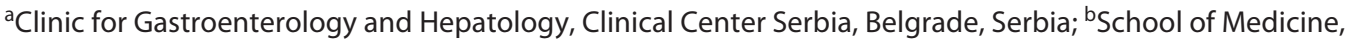 \\ University of Belgrade, Belgrade, Serbia
}

\section{Keywords}

Anemia $\cdot$ Primary care $\cdot$ Diagnostic algorithm

\begin{abstract}
Background: Anemia is a presentation of an underlying disease or deficiency. As stated by the WHO, anemia is defined as hemoglobin $(\mathrm{Hb})$ levels $<12.0 \mathrm{~g} / \mathrm{dL}$ in women and $<13.0 \mathrm{~g} / \mathrm{dL}$ in men. This review of clinical practice aimed to determine the diagnostic approach to anemia in primary care patients. Summary: Nutritional deficiencies, medications, chronic inflammatory conditions, malignancy, renal dysfunction, and bone marrow and inherent disorders contribute to anemia development. Anemia is classified and diagnosed by the values of hematological parameters, underlying pathological mechanism, and patient history. The diagnostic approach of anemia in primary care settings is focused on history, physical examination, laboratory findings including complete blood cell count, reticulocyte count, and peripheral smear examination, fecal occult blood test, and ultrasound findings. Key Messages: Anemia is the most common hematological disorder that represents a major health burden worldwide. Hb levels alter with gender, ethnicity, and physiological status. Anemia is often multifactorial. The evaluation of a patient with anemia in primary care includes clinical history, physical examination, and laboratory findings with fecal occult blood test and abdominal ultrasound. The wide variations in general practice in European
\end{abstract}

countries are based on different health care systems but also knowledge of GPs that reflect educational and research policy.

(c) 2021 S. Karger AG, Base

\section{Introduction}

Anemia is the most common hematological disorder that represents a major health burden worldwide affecting $>2$ billion people $[1,2]$. It is a presentation of an underlying condition, defined as a reduction in the proportion of the red blood cells (RBCs) [1-3]. A wide variety of factors contribute to the development of anemia including nutritional deficiencies, chronic disorders, inflammatory process, medications, malignancy, renal dysfunction, hereditary diseases, and bone marrow disorders [1,3]. Also, a higher prevalence of anemia has been associated with a number of medical conditions such as essential hypertension, congestive heart failure and coronary artery disease, hypothyroidism, and rheumatologic diseases [1].

According to the WHO, anemia is determined by hemoglobin $(\mathrm{Hb})$ levels $<13 \mathrm{~g} / \mathrm{dL}$ in adult males and $12 \mathrm{~g} /$ $\mathrm{dL}$ in females [4]. Nevertheless, Hb levels also alter with ethnicity and physiological status [1-3]. Due to the aging of the population, an increase of anemia has been registered in elderly people and recently defined by $\mathrm{Hb}$ levels 
$<12 \mathrm{~g} / \mathrm{dL}$ in both genders [2,3]. As stated by the WHO in 2016 , the estimated prevalence of anemia in women of childbearing age was $30.2 \%$ worldwide, while in Europe $20.2 \%$ [5]. Reduction of Hb levels in pregnancy as a result of physiological hemodilution is defined as pseudoanemia, maximal during 20-24 weeks of gestation [3,5]. Furthermore, increased odds of having anemia were determined in African Americans and Hispanic race compared to Caucasians $[1,2]$.

Apart from age, gender, and racial differences, anemia is recognized as an important risk factor to morbid mortality with serious impact on decreased quality of life, physical and mental capacities, psychiatric disorders, and susceptibility to infections [6-9]. Although the majority cases of anemia are diagnosed by general practitioners (GPs), studies about anemia management in primary care settings are still scarce across different European countries $[10,11]$. The wide variations in primary care units in European countries are based on different health care systems but also knowledge and awareness of GPs that reflect educational and research policy $[10,11]$.

\section{Classification and Diagnosis of Anemia}

The direct management of anemia depends on the underlying cause. The etiology-based approach is important in preventing, diagnosing, and treating anemia $[2,12]$. While acute anemia occurs predominantly due to acute blood loss or acute hemolysis, chronic anemia is more frequent and secondary to various causes [12].

Using a kinetic approach, anemias are classified based on the responsible pathophysiological mechanism including decreased RBC production, increased RBC destruction, and blood loss (shown in Fig. 1) [13]. The reticulocyte count is used to estimate the degree of effective erythropoiesis with corrected reticulocyte count $<2 \%$ in hypoproliferative anemia and $>2 \%$ in hyperproliferative anemia [2] According to the morphologic approach, anemias are categorized based on the size of RBC measured by mean corpuscular volume (MCV) into microcytic anemia (MCV $<80 \mathrm{fL}$ ), normocytic anemia (MCV 80-100 fL), and macrocytic anemia (MCV >100 fL) (shown in Fig. 2) [13].

The most common cause of anemia still remains iron deficiency $[2,6]$. The etiology of iron deficiency anemia (IDA) is variable and associated with a number of factors decreasing iron intake and absorption or increasing demand and loss. Various etiologies of IDA include a number of disorders including Helicobacter pylori infection, celiac disease, inflammatory bowel disease (IBD), atro-

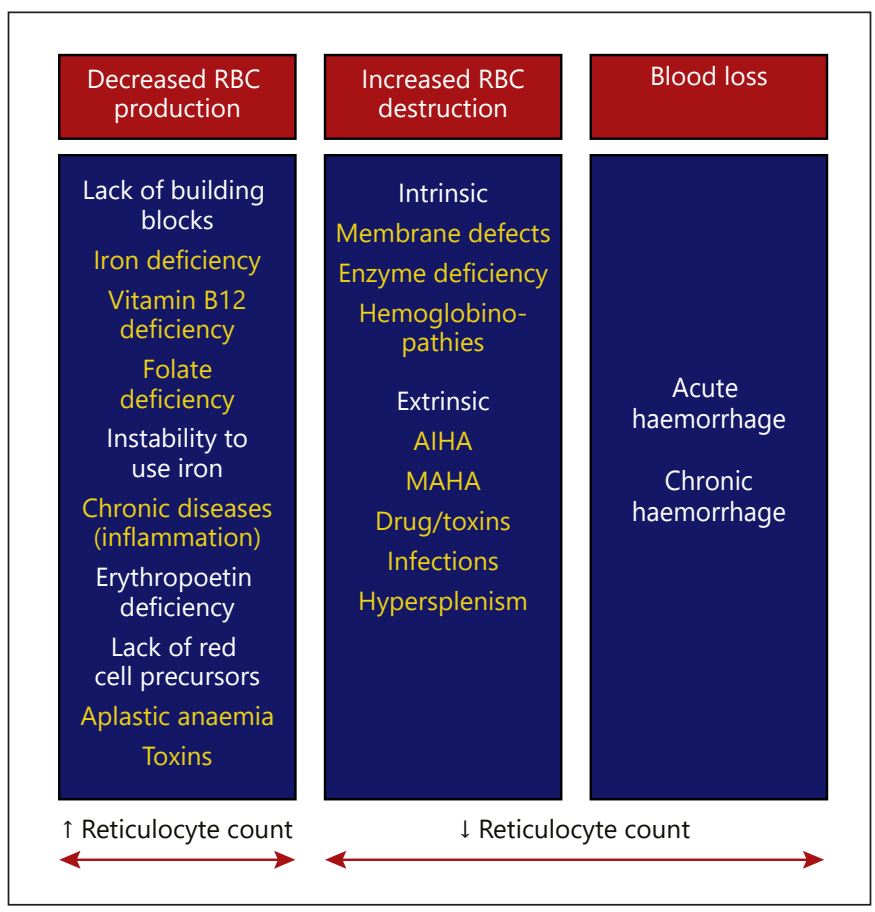

Fig. 1. Kinetic approach in classification of anemias. RBC, red blood cell.

phic gastritis due to long-term use of proton pump inhibitors, intoxication with heavy metals, or parasitic disease $[6,9,10]$.

\section{Clinical Presentation and Features of Anemia}

The manifestation of symptoms in patients depends on the etiology of anemia, severity of onset, and presence of other comorbidities. According to conducted investigations, the majority of patients become symptomatic when $\mathrm{Hb}$ values drop below $7.0 \mathrm{~g} / \mathrm{dL}[2,13,14]$. Obtaining a good history from a patient with anemia is important for further diagnostic algorithm with questions addressing prior blood loss, duration of anemia, associated features and comorbidities, and use of proton pump inhibitors, aspirin, and nonsteroidal anti-inflammatory drugs [15].

The evaluation of a patient includes a physical examination and presence of common signs of anemia such as pallor of the conjunctive and mucous membranes, fatigue, dizziness, dyspnea, tachycardia, and heart murmur $[15,16]$. Also, significant signs of anemia include cheilosis and glossitis indicating malnutrition (e.g., vitamin deficiency), jaundice, dark-colored urine due to hemolysis, hepatosplenomegaly, and bleeding (melena and hematuria) 
Fig. 2. Morphologic approach in classification of anemias. MCV, mean corpuscular volume.

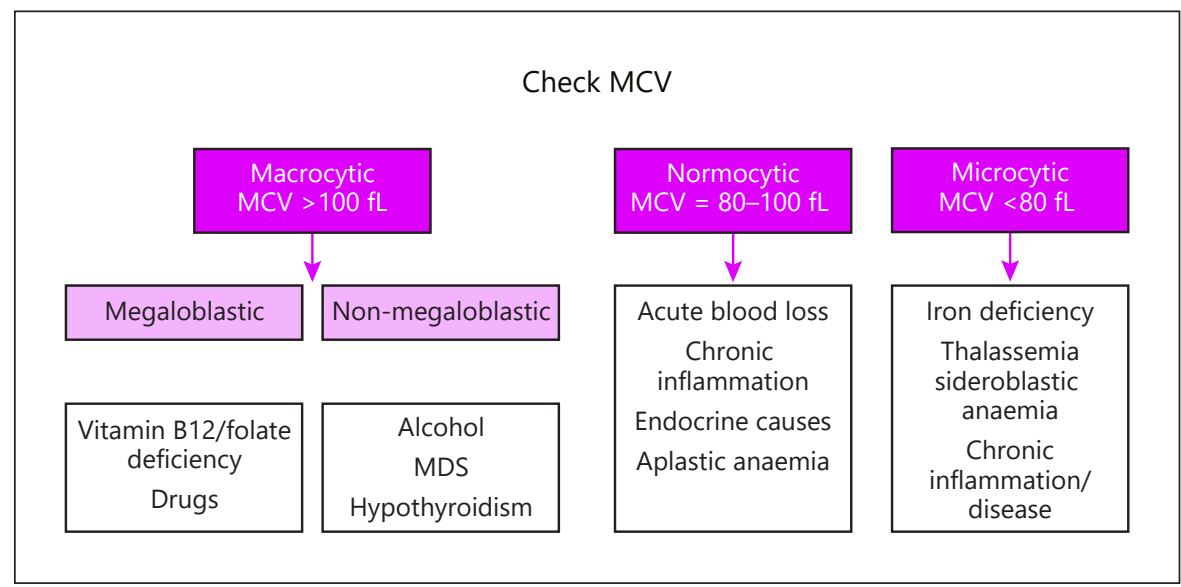

$[16,17]$. Patients with severe life-threatening anemia can experience sweating, thirst, cold extremities, and edema of lower limbs and develop respiratory distress and shock $[2,17]$. Nevertheless, very low Hb levels may be tolerated in the settings of slowly progressing anemia [18].

\section{Laboratory Assessment of Anemia}

The initial array of laboratory evaluation encompasses complete blood cell count with differential reticulocyte count, peripheral smear, and biochemical tests including iron profile (iron, ferritin, and TIBC), macrocytosis and hemolysis profile (vitamin B12, folate, methylmalonic acid, and homocysteine; haptoglobin, indirect bilirubin, and lactate dehydrogenase), serum creatinine, erythropoietin and estimated glomerular filtration rate, thyroid function tests and liver function tests, coagulation screen, and $\mathrm{Hb}$ electrophoresis $[15,18]$. IDA is the most common, estimated to be present in up to $2 \%$ of the adult population, and remains one of the top 5 leading causes of years lived with disability in humans $[19,20]$. Nevertheless, GPs have to be aware of overlapping conditions that can mask the macrocytosis expression of megaloblastic anemia. Approximately one-third of anemic elderly individuals have a nutritional basis for anemia with coexisting iron depletion and nutritional deficiency of folate or vitamin B12 $[4,14]$.

Fecal occult blood testing (FOBT) evaluates the presence of RBCs in the feces including guaiac FOBT (gFOBT) and fecal immunological test (FIT) [21]. The first fecal test used in colorectal carcinoma (CRC) screening was gFOBT. Although previous studies stated that an annual gFOBT can reduce incidence of CRC, the accuracy of this test is questionable [21]. The test is based on heme detection in the feces, which reacts with a hydrogen peroxidebased developer reagent to oxidize guaiac and turns blue [21]. Nevertheless, moderate concentrations of heme are needed to acquire color change. Furthermore, gFOBT depends on the pure oxidation process while influence of peroxidases in food can lead to false-positive results [21, 22]. Also, administration of antioxidants such as vitamin $\mathrm{C}$ can result in false negativity of gFOBT [21]. Therefore, food restrictions including red meat and plants with peroxidases must be implemented before the gFOBT. Limitations of the gFOBT are shown in Table 1.

The FIT assesses the quantity of $\mathrm{Hb}$ in the feces. In recent years, FIT has been endorsed by the UK's National Institute for Health and Care Excellence (NICE) guideline for CRC screening [21]. Also, the World Endoscopy Organization Expert Working Group recommended FIT as the preferred technology compared to gFOBT because there is no influence by other fecal constituents, including medication and dietary products [23]. According to recent data, FIT has greater values than gFOBT in detecting neoplasms in the lower gastrointestinal tract. Namely, the 3-sample FIT in high-risk patients could improve the specificity of FIT by $35 \%$ compared with a single test $[21,24]$.

Nevertheless, broad implementation of FOBT in all low-risk symptomatic patients can lead to endoscopy capacity crisis due to patients with false-positive results or delayed diagnosis for patients with false-negative tests [25]. According to the NICE diagnostic guideline from 2017, fecal blood testing should be performed in patients without rectal bleeding who have unexplained symptoms, but do not meet criteria for suspected cancer [26]. Hopkins et al. [27] reported microcytosis as a predictor 
Table 1. Limitations of gFOBT

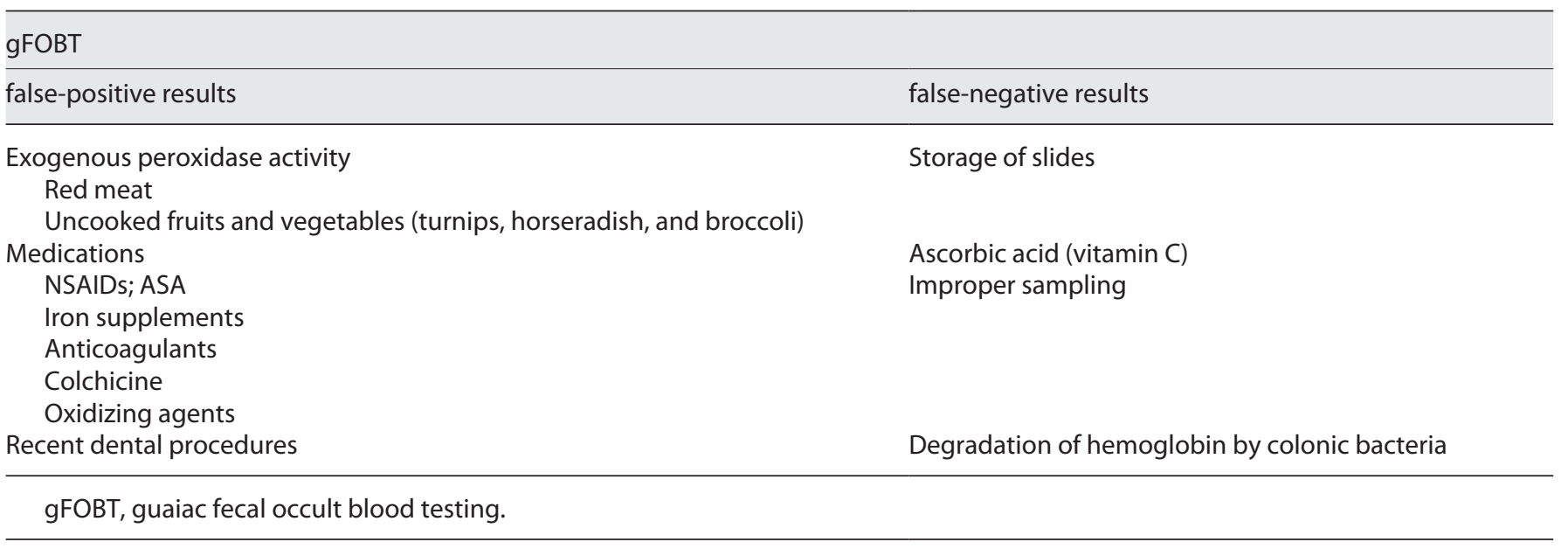

of underlying cancer even in cases when $\mathrm{Hb}$ is normal, especially in males.

Individuals with positive FOBT must be referred for further investigations. According to conducted data, the availability of open-access endoscopy ranged from $28 \%$ in Poland to over $80 \%$ in Holland, Czech, and the UK [10]. Also, $92 \%$ of Czech respondents, $52 \%$ in Spain, $45 \%$ in the Netherlands, $42 \%$ in Greece, $27 \%$ in the UK, and 21\% in Poland reported open access to colonoscopy [10].

\section{Rectal Bleeding in General Practice}

The reported GP approach to patients with low-risk rectal bleeding, below the age of 50 and presenting without alarm signals, includes rectal and abdominal examination [10]. Nevertheless, patients with rectal bleeding associated with alarm signals were referred to endoscopic examination, with reported $95 \%$ of GPs in the UK and $70 \%$ in Poland using a special referral arrangement that provides a specialist appointment within 2 weeks [10]. While GPs from Spain, Holland, Poland, and Czech would make an urgent referral in $30-50 \%$ of cases, up to $60 \%$ would arrange a colonoscopy [10]. Also, the awareness of the national guideline for colorectal cancer risk differs between countries, from $29 \%$ in the Netherlands to $94 \%$ in the Czech Republic [10]. Colorectal cancer is often mistaken for hemorrhoids due to similarity of clinical manifestations, causing delayed diagnosis and ineffective treatments. The high awareness of CRC screening guidelines in some countries is based on established screening programs in these countries. While Portugal, Spain, Belgium, the United Kingdom, the Netherlands,
Denmark, Slovenia, Lithuania, Estonia, and Finland have a strong primary care system, relatively weak primary care organization is reported in countries of central and south-eastern Europe and Turkey [11].

\section{Abdominal US in Anemia Diagnostics}

In the primary care settings, ultrasound (US) of the abdomen as an essential noninvasive diagnostic approach can detect possible alterations such as abdominal masses that can address the possible etiology of detected anemia. The US findings of the liver, spleen, bowel, stomach, and gynecologic or lymph node alterations can guide further investigation in patients with anemia [28]. However, the role of abdominal US in evaluation of anemia is inferior to endoscopic examination including upper and lower gastrointestinal endoscopy.

According to Focused Assessment with Sonography for Trauma (FAST), rapid detection of free fluid can be suggestive for hemoperitoneum, hemothorax, and/or hemopericardium in patients with anemia due to acute blood loss [28]. The use of FAST has been reported to decrease the abdominal CT use by approximately $50 \%$ $[28,29]$. The early detection of bleeding in nontraumatic cases is based on sonographic markers of hypovolemia including the diameter of the inferior cava vein and the thickness of the left ventricle [28].

The use of US in patients with anemia due to intestinal diseases can be helpful. Namely, the European Crohn's and Colitis Organization (ECCO) guidelines highlighted ultrasonography as the imaging technique of choice for screening IBD patients [30]. While ultrasonographic signs of 
thickening, decreased compressibility, and increased vascularization of the bowel wall can be detected in IBD, "pseudo-kidney sign" (hypoechoic bowel wall thickening and irregular contour, loss of stratification of the wall layers, and the absence of normal peristalsis) can be suggestive of CRC [28, 31]. Detection of liver lesions suggesting metastasis can indicate presence of malignancy [28, 31]. The US findings in lymphoproliferative disorders presented with anemia include splenomegaly and enlarged abdominal lymph nodes [28]. In patients with clinical signs of maldigestion and anemia, US may identify the radiological signs of chronic pancreatitis (reduction in the size of the pancreas, irregular profiles, parenchymal calcifications, or dilated Wirsung duct with stones) [32].

\section{Conclusion}

GPs are the first contact of the patient with the health care system. In addition to the limited research evidence from primary care units, the scarce recommendations for anemia may also be driven by concerns about overwhelmed endoscopy services by referrals of patients or local priorities and beliefs about the costs and benefits of diagnostic testing.

\section{Conflict of Interest Statement}

The authors have no conflicts of interest to declare.

\section{Funding Sources}

There is no relevant funding source or sponsor to this review article to declare.

\section{Author Contributions}

Tamara Milovanovic wrote and designed the review article; Sanja Dragasevic wrote the article and performed the review of the literature; Andreja Nebojsa Nikolic, Milica Stojkovic Lalosevic, and Dusan D. Popovic analyzed current novelty data; Aleksandra Pavlovic-Markovic and Miodrag N. Krstic provided guidance in this research.

\section{References}

1 Gandhi SJ, Hagans I, Nathan K, Hunter K, Roy S. Prevalence, comorbidity and investigation of anemia in the primary care office. J Clin Med Res. 2017;9:970-80.

2 Turner J, Parsi M, Badireddy M. Anemia. StatPearls. Treasure Island (FL): StatPearls Publishing; 2020 Sep 10.

3 Cappellini MD, Motta I. Anemia in clinical practice-definition and classification: does hemoglobin change with aging? Semin Hematol. 2015;52:261-9.

4 World Health Organization. Haemoglobin concentrations for the diagnosis of anaemia and assessment of severity. Vitamin and mineral nutrition information system. Geneva: World Health Organization; 2011.

5 Bayen S, Le Grand C, Bayen M, Richard F, Messaadi N. Anemia management in nonmenopausal women in a primary care setting: a prospective evaluation of clinical practice. BMC Fam Pract. 2020;21:13.

6 Cook RL, O'Dwyer NJ, Parker HM, Donges CE, Cheng HL, Steinbeck KS, et al. Iron deficiency anemia, not iron deficiency, is associated with reduced attention in healthy young women. Nutrients. 2017;9(11):1216.

$7 \mathrm{Xu} \mathrm{F}$, Roberts L, Binns C, Sullivan E, Homer CSE. Anemia and depression before and after birth: a cohort study based on linked population data. BMC Psychiatry. 2018;18:224.

8 de Pontual L. Iron and susceptibility to infections. Arch Pediatr. 2017;24:5S14-7.
9 Kwaan HC. Infection and anemia. Infect Disord Drug Targets. 2011;11(1):40-4.

10 Seifert B, Rubin G, de Wit N, Lionis C, Hall $\mathrm{N}$, Hungin $\mathrm{P}$, et al. The management of common gastrointestinal disorders in general practice A survey by the European Society for Primary Care Gastroenterology (ESPCG) in six European countries. Dig Liver Dis. 2008;40:659-66.

11 Kringos DS, Boerma WGW, Hutchinson A, Saltman RB. Building primary care in a changing Europe. Copenhagen (Denmark): European Observatory on Health Systems and Policies; 2015.

12 Badireddy M, Baradhi KM. Chronic Anemia. StatPearls. Treasure Island (FL): StatPearls Publishing; 2020 Nov 18.

13 Kundrapu S, Noguez J. Laboratory assessment of anemia. Adv Clin Chem. 2018;83: 197-225.

14 Lanier JB, Park JJ, Callahan RC. Anemia in older adults. Am Fam Physician. 2018;98:437-42.

15 Powell DJ, Achebe MO. Anemia for the primary care physician. Prim Care. 2016;43:527-42.

16 De Franceschi L, Iolascon A, Taher A, Cappellini MD. Clinical management of iron deficiency anemia in adults: systemic review on advances in diagnosis and treatment. Eur J Intern Med. 2017;42:16-23.

17 Long B, Koyfman A. Emergency medicine evaluation and management of anemia. Emerg Med Clin North Am. 2018;36:609-30.
18 Freeman AM, Rai M, Morando DW. Anemia screening. StatPearls. Treasure Island (FL): StatPearls Publishing; 2020 Aug 4.

19 Cappellini MD, Musallam KM, Taher AT. Iron deficiency anaemia revisited. J Intern Med. 2020;287:153-70.

20 Ko CW, Siddique SM, Patel A, Harris A, Sul$\tan$ S, Altayar O, et al. AGA clinical practice guidelines on the gastrointestinal evaluation of iron deficiency anemia. Gastroenterology. 2020;159:1085-94.

21 Li JN, Yuan SY. Fecal occult blood test in colorectal cancer screening. J Dig Dis. 2019; 20:62-4.

22 Carroll MR, Seaman HE, Halloran SP. Tests and investigations for colorectal cancer screening. Clin Biochem. 2014;47:921-39.

23 Fraser CG, Allison JE, Halloran SP, Young GP. Expert working group on fecal immunochemical tests for hemoglobin, colorectal cancer screening committee, world endoscopy organization. A proposal to standardize reporting units for fecal immunochemical tests for hemoglobin. J Natl Cancer Inst. 2012;104: $810-4$.

24 Wu D, Luo HQ, Zhou WX, Qian JM, Li JN The performance of threesample qualitative immunochemical fecal test to detect colorectal adenoma and cancer in gastrointestinal outpatients: an observational study. PLoS One. 2014;9:e106648. 
25 D'Souza N, Brzezicki A, Abulafi M. Faecal immunochemical testing in general practice. $\mathrm{Br}$ J Gen Pract. 2019;69:60-1.

26 National Institute for Health and Care Excellence. Quantitative faecal immunochemical tests to guide referral for colorectal cancer in primary care. London: NICE; 2017.

27 Hopkins R, Bailey SE, Hamilton WT, Shephard EA. Microcytosis as a risk marker of cancer in primary care: a cohort study using electronic patient records. Br J Gen Pract. 2020; 70:e457-62.
28 Mozzini C, Pesce G, Casadei A, Girelli D, Soresi M. Ultrasound as first line step in anaemia diagnostics. Mediterr J Hematol Infect Dis. 2019;11:e2019066.

29 Stengel D, Bauwens K, Rademacher G, Ekkernkamp A, Güthoff C. Emergency ultrasound-based algorithms for diagnosing blunt abdominal trauma. Cochrane Database Syst Rev. 2013;7:CD004446.

30 Gomollón F, Dignass A, Annese V 3rd. European evidence-based consensus on the diagnosis and management of Crohn's dis- ease 2016: part 1: diagnosis and medical management. J Crohn Colitis. 2017;11:325.

31 Zhang F, Miao LY, Ge HY, Tan S, Li ZQ, Zhao B. Usefulness of contrast-enhanced ultrasound in differentiating inflammatory Bowel disease from colon cancer. Ultrasound Med Biol. 2018;44(1):124-33.

32 Frulloni L, Falconi M, Gabbrielli A, Gaia E, Graziani R, Pezzilli R, et al. Italian consensus guidelines for chronic pancreatitis. Dig Liver Dis. 2010;42:S381-406. 